\title{
SPARSITY ANALYSIS OF THE
}

QR FACTORIZATION

D. Hare, C.R. Johmsot, D.D. OLestry

AND

P. VAI DEI DRIESSCHE

WARCH 1990

DHS-536-IR

REVISED : NOVEMBER 1991 
by

Donovan R. Hare

Department of Mathematics and Statistics

University of Victoria

Victoria, British Columbia

Canada V8V 3P4

\author{
Charles R. Johnson ${ }^{(1)}$ \\ Department of Mathematics \\ College of Villiam and Mary \\ Villiamsburg, VA 23185 \\ U.S.A. \\ D.D. 01 esky $(2)$ \\ Department of Computer Science \\ University of Victoria \\ Victoria, British Columbia \\ Canada V8W 3P6
}

and

P. van den Driessche ${ }^{(3)}$

Department of Mathematics and Statistics

University of Victoria

Victoria, British Columbia

Canada V8V 3P4

(1) The work of this author was supported in part by the National Science Foundation grant DMS90-00839 and Dffice of Naval Research Contract N00014-90-J-1739.

(2) The work of this author was supported in part by NSERC grant A-8214 and by the University of Victoria Committee on Faculty Research and Travel.

(3) The work of this author was supported in part by NSERC grant A-8965 and by the University of Victoria Committee on Faculty Research and Travel. 


\section{Sparsity Analysis of the QR Factorization}

\section{Abstract}

Given only the zero-nonzero pattern of an $m-b y-n$ matrix $A$ of full column rank, which entries of $Q$ and which entries of $R$ in its $Q R$ factorization must be zero and which entries may be nonzero? We give a complete answer to this question, which involves an interesting interplay between combinatorial structure and the algebra implicit in orthogonality. To this end some new sparse structural concepts are introduced, and an algorithm to determine the structure of $Q$ is given. The structure of $R$ then follows immediately from that of $Q$ and $A$. The computable zero/nonzero structures for the matrices $Q$ and $R$ are proven to be tight, and the conditions on the pattern for $A$ are the weakest possible (namely that it allows matrices A with full column rank). This complements existing work that focussed upon $R$ and then only under an additional combinatorial assumption (the strong Hall property).

Key words: QR factorization, bipartite graph, Hall property, sparse matrix, combinatorial matrix theory, orthogonality. 
1. Introduction.

For $m \geq n$, an $m-b y-n$ matrix $A=\left[a_{i j}\right]$ has a unique factorization $A=Q R$, in which $Q=\left[q_{i j}\right]$ is $m-b y-n$ and has orthonormal columns and $R=\left[r_{i j}\right]$ is $n-b y-n$ and upper triangular with positive diagonal entries, exactly when $A$ has full rank, $n$. In this event we say that $A$ has a unique $Q R$ factorization, and we mean the one in which $R$ has positive diagonal. There are several possible ways to compute such a factorization (with exact arithmetic), for example, using plane rotations (method of Givens), Householder transformations, or the Gram-Schmidt procedure. When A has a unique $Q R$ factorization, it does not matter, except for insight or computational considerations, which algorithm is used to obtain the factorization.

By a nonzero pattern $\mathscr{b}$ of size m-by- $n$ we mean the set of positions in an $\mathrm{m}-\mathrm{by}-\mathrm{n}$ matrix in which the nonzero entries occur. We typically describe such a pattern via an $\mathrm{m}-\mathrm{by}-\mathrm{n}$ array with 0 and $*$ entries, in which * denotes a nonzero. For example $A=\left[\begin{array}{rr}-3 & 0 \\ 1 & 2 \\ 0 & \pi\end{array}\right]$ is a matrix with pattern $\mathscr{C}=\left[\begin{array}{ll}* & 0 \\ * & * \\ 0 & *\end{array}\right]$. If matrix A has pattern $\mathscr{C}$, then we write $A \in \mathscr{C}$. By $\mathscr{b}+\mathscr{B}$ (or $\mathscr{b} \cup \mathscr{A}$ ) we mean the union of two patterns of the same size, that is, a pattern that has a zero in a given position only when both patterns are zero in that position. Multiplication of patterns is carried out under the following assumptions: $\left(*^{*} \cdot *^{*}\right)=*$ and $\left(*^{*} \cdot \hat{0}\right)=(\hat{0} \cdot *)=(0 \cdot \hat{0})=0$. Two column vectors (or vector patterns) are combinatorially orthogonal if each term in the inner product is zero.

Our primary purpose is to give a complete solution to the following problem. For $m \geq n$, given an $m-b y-n$ nonzero pattern to that allows full rank, determine the union, over all full column rank matrices $A \in \mathscr{K}$, of all 
patterns occurring in the matrix $Q$ and in the matrix $R$ such that $A=Q R$ is the $Q R$ factorization of $A$. The resulting unions are denoted by 2 and $\Re$, respectively. Our solution to this problem continues earlier work [GH, GLN, CEG, and a small part of GN] that focussed mainly upon the upper triangular pattern $\mathscr{R}$ (rather than 2) and gave a description only under an additional combinatorial assumption (the strong Hall property) on the pattern 6. The present work is based on the Gram-Schmidt procedure and is in the spirit of an analogous combinatorial analysis of the $L U$ factorization of a square matrix that was given in [JOD]. However, there are subtleties that are quite different from the $L U$ case.

We note that, for a given pattern $\mathscr{b}$, different zero patterns may occur among the Q's, or among the R's, in the QR factorization of different full rank matrices A with pattern $\mathscr{t}_{0}$. This is due to the possibility of chance numeric relations among the entries of a specific matrix A which cause "accidental zeros". Our interest is in ignoring chance cancellation and in obtaining the union of all patterns for both $Q$ and $R$. Knowledge of 2 and $\mathscr{R}$ is useful for computational purposes as they are the smallest patterns that are guaranteed to contain the nonzero entries of the $Q \mathbb{R}$ factorization of an arbitrary $A \in \mathscr{b}$. Thus $\mathcal{L}$ and $R$ could be used to define a suitable data structure for $Q$ and $R$, respectively.

Some particularly simple cases occur when $\mathscr{t}$ is square. If $\mathscr{t}$ is full upper triangular, then $\lambda$ has the same pattern and 2 is diagonal. If $\mathscr{b}$ is full upper Hessenberg, then 2 has this same pattern and $\mathscr{R}$ is full. However, if $\mathscr{t}$ is full lower triangular, then both $\mathscr{R}$ and $\mathcal{L}$ are full.

A combinatorial necessary condition for an m-by-n matrix $A$ to have rank $\mathbf{n}$ is the following (see, e.g. [CEG]). 
Definition. For $m \geq n$, an $m-b y-n$ matrix $A$ (or pattern $\mathscr{b}$ ) satisfies the Hall property if every $k$ columns, $1 \leq k \leq n$, collectively have nonzero entries in at least $k$ rows.

It is clear that there are full rank matrices with pattern $\mathscr{t}$ if and only if $\mathscr{6}$ satisfies the Hall property. So that our problem be well-defined, we wish to assume that each matrix $A$ to be factored has a unique $Q R$ factorization. Thus, the problem statement assumed that $A$ has full column rank, and therefore, we restrict our attention to patterns satisfying the Hall property. If $A$ has a unique $Q R$ factorization $A=Q R$, then, for an $m-b y-m$ permutation matrix $P, P A$ also has a unique $Q R$ factorization $P A=(P Q) R$. Since $Q$ is modified in this simple way, and $R$ not at all, we may permute the rows of A for convenience. For example, under the Hall property assumption, we may permute the rows so that the diagonal entries are all nonzero. We note, however, that permutation of the columns of $A$, in general, radically changes both $Q$ and $R$.

In [CEG] the possible nonzeros for $\mathscr{R}$ are identified under an additional assumption. (The following definition, a slight variant from what appears in [CEG], now seems to be standard.)

Definition. For $m \geq n$, an $m-b y-n$ matrix $A$ (or pattern 6 ) satisfies the strong $\underline{\text { Hall property }}$ if

(i) $\mathrm{m}=\mathrm{n}>1$ and every $\mathrm{k}$ columns, $1 \leq \mathrm{k}<\mathrm{n}$, collectively have nonzero entries in more than $k$ rows, or

(ii) $\mathrm{m}>\mathrm{n}$ and every $\mathrm{k}$ columns, $1 \leq \mathrm{k} \leq \mathrm{n}$, collectively have nonzero entries in more than $k$ rows. 
Note that, if $m=n$ and each main diagonal entry of $A$ is nonzero, then $A$ satisfies the strong Hall property if and only if $A$ is irreducible. Under the strong Hall assumption on $\mathscr{b}$ it is shown in [CEG] that $\mathscr{R}$ is accurately predicted by applying "symbolic Gaussian elimination" (i.e. "symbolic LU factorization") to the symbolic product $\mathscr{C}^{\mathrm{T}} \mathscr{b}$, or by doing "symbolic Givens rotations" on $\mathscr{6}$. In this case, $\Omega$ can also be bounded by the pattern of $\mathrm{U}$ in the symbolic LU factorization (with partial pivoting) of $\mathscr{b}$, see $[\mathrm{GN}]$.

Without the strong Hall assumption, the complete analysis is considerably more delicate. There can be interplay between the combinatorics of the arrangement of zeros and nonzeros and the algebra (associated with orthogonality required in $Q$ ) of the $Q R$ calculation that forces zeros that are missed by any naive analysis. This is illustrated in the following example from [CEG], in which $\mathscr{6}$ satisfies the Hall property but not the strong Hall property.

Example (1.1). Let

$$
\mathscr{b}=\left[\begin{array}{llllll}
* & 0 & 0 & 0 & 0 & 0 \\
* & * & * & 0 & 0 & 0 \\
0 & 0 & * & * & * & * \\
0 & 0 & 0 & * & 0 & 0 \\
0 & 0 & 0 & 0 & * & 0 \\
* & 0 & 0 & 0 & 0 & *
\end{array}\right] .
$$

As pointed out in [Ge], when 2 and $\mathscr{R}$ are predicted by a symbolic analog of the (numeric) method of Givens (where nonzero entries in the jth column are zeroed out in the order $a_{j+1, j}$ to $a_{n, j}$ ), too much fill is predicted. Specifically this approach gives 


$$
\mathcal{L}=\left[\begin{array}{llllll}
* & * & \otimes & \otimes & \otimes & * \\
* & * & \otimes & \otimes & \otimes & \otimes \\
0 & 0 & * & \otimes & \otimes & \otimes \\
0 & 0 & 0 & * & \otimes & \otimes \\
0 & 0 & 0 & 0 & * & \otimes \\
* & * & \otimes & \otimes & \otimes & *
\end{array}\right], \quad R=\left[\begin{array}{llllll}
* & * & * & 0 & 0 & * \\
& * & * & 0 & 0 & * \\
& * & * & * & * \\
& & * & \otimes & \otimes \\
0 & & & * & \otimes \\
& & & & & *
\end{array}\right]
$$

where $\otimes=^{*}$, whereas $\otimes$ gives the true patterns. The entries in $\mathbb{R}$ designated by are called "bogus fill" in [CEG]. The pattern for $\mathscr{R}$ in (1.2) with $\otimes=*$ is also obtained when symbolic LU factorization of the symbolic product $\mathscr{C}_{\mathscr{C}}^{T}$ is performed [GH] or when symbolic LU factorization (with partial pivoting) of $\mathscr{b}$ is performed [GN].

In the next section, we begin by defining the notion of a Hall set. This enables us to define a sequence of bipartite graphs that lead to the identification of all zero entries in 2. The orthogonality of $Q$ can require zero entries in 2 in somewhat subtle ways. Some graph-theoretic results concerning these bipartite graphs are given in section 3 . The purpose of section 4 is to show that the remaining entries of 2 are, in fact, nonzeros. It begins with explicit consideration of a special form for the pattern of $\mathscr{t}$ and then uses embedding and continuity to prove our result for general patterns. An algorithm for determining 2 is given. In section 5 it is shown how to determine the pattern $\mathscr{R}$ in a simple way, once the pattern 2 is known. 


\section{Zero Entries in 2.}

Related to the Hall properties, we introduce the concept of a Hall set. Given any m-by-n matrix A (or pattern f) we denote the jth column of A (or $\mathscr{A}$ ) by $A_{j}$ (or $\mathscr{C}_{j}$ ), and the restriction of $A_{j}$ to the row index set $a$ by $\mathrm{A}_{\mathrm{j}}[a]$.

Definition. For an $m-b y-n$ matrix $A$ (or pattern 6 ) with $m \geq n$, a set of $\mathrm{k}$ columns in $\mathrm{A}$ ( or 10$), 1 \leq \mathrm{k} \leq \mathrm{n}$, is a $\underline{\mathrm{Hall}}$ set if these columns collectively have nonzero entries in exactly $k$ rows.

Under the assumption that A (or $\mathscr{6}$ ) has the Hall property, the union of two Hall sets is a Hall set, so there exists a unique Hall set of maximum cardinality $(\geq 0)$ in any given set of columns. Let $S_{j}$ be the Hall set of maximum cardinality in the first $j$ columns; we define $S_{0}=\phi$. For example, in the pattern $(1.1), S_{1}=\phi$ and $S_{j}=\left\{\mathscr{c}_{2}, \ldots, \mathscr{c}_{j}\right\}$ for $2 \leq j \leq 5$. Note that if an $m-b y-n$ matrix has the strong Hall property, then $S_{j}=\phi$,

$1 \leq \mathrm{j} \leq \mathrm{n}-1$.

The Gram-Schmidt orthonormalization process (see, e.g. [HJ, 0.6.4]) can be used to construct the matrix $Q$ of the $Q R$ factorization of a matrix $A$. We show below how to adapt the Gram-Schmidt procedure to determine 2 for a pattern $\mathscr{C}$ Clearly $\mathscr{q}_{1}=\mathscr{G}_{1}$ as $Q_{1}$ is a (nonzero) multiple of $A_{1}$. If $\mathscr{h}_{2}$ is combinatorially orthogonal to $\hbar_{1}$, then $2_{2}=\hbar_{2}$. However, if $\hbar_{2}$ is not combinatorially orthogonal to $\mathscr{c}_{1}$ and $S_{1}=\phi$, then $\mathscr{2}_{2}=\mathscr{h}_{1}+\mathfrak{K}_{2}=\mathscr{2}_{1}+\mathfrak{h}_{2}$. The effect of Hall sets on this columnise "naive accumulation" of nonzero entries is now investigated.

Let $s_{j}$ be the set of all row indices covered by the columns of $s_{j}$, thus $\left|S_{j}\right|=\left|s_{j}\right|$. (Note that if we assume all diagonal entries of $\mathscr{b}$ are 
*, then $s_{j}=\left\{i: 1 \leq i \leq j, \mathscr{c}_{i} \in S_{j}\right\}$; however, we do not make this assumption in this section.) For a given $\mathscr{b}$, we define a bipartite graph that leads to a partition of the row indices of $\mathscr{b}$ and identifies the zero entries in a column of 2. For a fixed $j, 1 \leq j \leq n$, the bipartite graph $\mathrm{B}_{\mathrm{j}}(\mathscr{C})=\left(\mathrm{R}_{\mathrm{j}}(\mathscr{C}), \mathrm{C}_{\mathrm{j}}(\mathscr{O}) ; \mathrm{E}_{\mathrm{j}}(\mathscr{C})\right)$, is defined as follows:

$$
\begin{aligned}
& \mathrm{C}_{\mathrm{j}}(\mathscr{\mathscr { C }})=\left\{\mathrm{c}_{\mathrm{k}}=\mathrm{k}: 1 \leq \mathrm{k} \leq \mathrm{j} \text { and } \mathscr{C}_{\mathrm{k}} \notin \mathrm{s}_{\mathrm{j}-1}\right\} ; \\
& \mathrm{R}_{\mathrm{j}}(\mathscr{\mathscr { C }})=\left\{\mathrm{r}_{\mathrm{i}}=\mathrm{i}: 1 \leq \mathrm{i} \leq \mathrm{m} \text { and there exists } \mathrm{c}_{\mathrm{k}} \in \mathrm{C}_{\mathrm{j}}(\mathscr{\mathscr { C }}) \text { with } \mathrm{a} *\right. \text { in } \\
&\text { row } \left.\mathrm{r}_{\mathrm{i}} \text { and } \mathrm{r}_{\mathrm{i}} \notin \mathrm{s}_{\mathrm{j}-1}\right\} ; \\
&\left\{\mathrm{r}_{\mathrm{i}}, \mathrm{c}_{\mathrm{k}}\right\} \in \mathrm{E}_{\mathrm{j}}(\mathscr{\mathscr { C }}) \text {, the undirected edge set, if and only if } \mathrm{r}_{\mathrm{i}} \in \mathrm{R}_{\mathrm{j}}(\mathscr{\mathscr { C }}) \\
& \text { and } \mathrm{c}_{\mathrm{k}} \in \mathrm{C}_{\mathrm{j}}(\mathscr{\mathscr { O }}) \text { and the }(\mathrm{i}, \mathrm{k}) \text { position of } \mathscr{\mathscr { C }} \text { is } *
\end{aligned}
$$

Thus, to construct $B_{j}(\mathscr{O})$, delete columns in $S_{j-1}$, rows $s_{j-1}$ and zero rows from $\left[\mathscr{c}_{1}, \ldots, \mathscr{c}_{j}\right]$ and take the bipartite graph of the remaining pattern (with the labelling inherited from the original pattern).

A path (undirected) in $B_{j}(\mathscr{A})$ is an alternating sequence of row and column vertices of the form $r_{k_{1}}, c_{j_{1}}, r_{k_{2}}, \ldots, r_{k_{i}}, c_{j_{i}}$ with each edge $\left\{\mathrm{r}_{\mathrm{k}_{1}}, \mathrm{c}_{\mathrm{j}_{1}}\right\},\left\{\mathrm{c}_{\mathrm{j}_{1}}, \mathrm{r}_{\mathrm{k}_{2}}\right\}, \ldots,\left\{\mathrm{r}_{\mathrm{k}_{\mathrm{i}}}, \mathrm{c}_{\mathrm{j}_{\mathrm{i}}}\right\} \in \mathrm{E}_{\mathrm{j}}(\mathscr{G})$. Such a path is associated with the sequence $\left(k_{1}, j_{1}\right),\left(k_{2}, j_{1}\right), \ldots,\left(k_{i}, j_{i}\right)$ of entries that are * in 6. A path may begin or end with either a row or column vertex, may have just one vertex, and is always assumed to be simple.

Let $p_{j}$ be the set of all row indices occurring in $R_{j}(\mathscr{6})$ that can be reached by a path from $c_{j}$. Let $u_{j}$ be the set of all row indices occurring in $R_{j}(\mathscr{G})$ that cannot be reached by a path from $c_{j}$. Finally, let $t_{j}$ be 
the set of all row indices of $\mathscr{t}$ not in $\mathrm{R}_{\mathrm{j}}(\mathscr{b})$ or $\mathrm{s}_{\mathrm{j}-1}$. We then have that $s_{j-1}, p_{j}, u_{j}$ and $t_{j}$ form a partition of all $m$ row indices. To illustrate this partition, consider again the pattern $\mathscr{C}$ in example (1.1); the bipartite graphs for $j=3$ and for $j=6$ are shown in Figure 1 .

The bipartite graph $\mathrm{B}_{3}(\mathscr{6})$
$s_{2}=\left\{r_{2}\right\}$

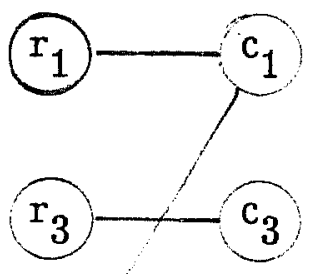
$\mathrm{u}_{3}=\left\{\mathrm{r}_{1}, \mathrm{r}_{6}\right\}$
$\mathrm{t}_{3}=\left\{\mathrm{r}_{4}, \mathrm{r}_{5}\right\}$
$\mathrm{p}_{3}=\left\{\mathrm{r}_{3}\right\}$
$\mathrm{p}_{6}=\left\{\mathrm{r}_{1}, \mathrm{r}_{6}\right\}$

$$
\begin{aligned}
& \mathrm{s}_{5}=\left\{\mathrm{r}_{2}, \mathrm{r}_{3}, \mathrm{r}_{4}, \mathrm{r}_{5}\right\} \\
& \mathrm{p}_{6}=\left\{\mathrm{r}_{1}, \mathrm{r}_{6}\right\} \\
& \mathrm{u}_{6}=\phi \\
& \mathrm{t}_{6}=\phi
\end{aligned}
$$$$
6=\left\{r_{1}, r_{6}\right\}
$$

The bipartite graph $\mathrm{B}_{6}(\mathscr{b})$$$
\begin{aligned}
& \mathrm{r}_{1}-\mathrm{c}_{1} \\
& \mathrm{r}_{6}-\mathrm{c}_{6}
\end{aligned}
$$

Figure 1.

We use the partition defined above to identify the zero entries in a column of 2 .

Theorem 2.1: For in $\geq$ ñ, let $\mathscr{b}$ be an m-by-ñ pattern with tile ilall property. For any fixed $j, 1 \leq j \leq n$, the entries of $2_{j}$ in positions $s_{j-1}, t_{j}$ and $u_{j}$ are zero. 
Proof: We rely on two facts that follow from the Gram-Schmidt procedure:

(i) $q_{j} \in \operatorname{span}\left\{A_{1}, \ldots, A_{j}\right\}$, and

(ii) $Q_{j}$ is orthogonal to $A_{1}, \ldots, A_{j-1}$.

Consider first the positions $t_{j}$. In these rows the entries in columns $A_{1}, \ldots, A_{j}$ of any $A \in \mathscr{C}$ are all zero; so by (i), the positions $t_{j}$ are all zero for any $Q_{j} \in 2_{j}$.

For the positions $s_{j-1}$, fact (ii) above implies that $Q_{j}$ is orthogonal to each column in $s_{j-1}$. But the columns of $s_{j-1}$, restricted to rows $s_{j-1}$, span a subspace of dimension $\left|s_{j-1}\right|$ and are zero outside these rows (by the definition of a Hall set). Thus $Q_{j}\left[s_{j-1}\right]$ is orthogonal to every vector in the above subspace, and so must be zero. Thus the positions $s_{j-1}$ of any $q_{j} \in \mathcal{Q}_{j}$ are zero; these are due to the presence of a Hall set. Finally consider the positions $u_{j}$, and let $\left\{A_{i_{1}}, \ldots, A_{i_{k}}\right\}$ be the set of columns on a path in $B_{j}(\mathscr{A})$ from indices in $\mathbf{u}_{j}$. From $(i)$ and because $u_{j} \cap\left(p_{j} \cup s_{j-1}\right)=\phi, q_{j}\left[u_{j}\right] \in \operatorname{span}\left\{A_{i_{1}}\left[u_{j}\right], \ldots, A_{i_{k}}\left[u_{j}\right]\right\}$. Because of (ii), and because $\mathrm{A}_{\mathrm{i}_{\mathrm{g}}}\left[\mathrm{p}_{\mathbf{j}}\right]=0, \mathrm{~g}=1, \ldots, \mathrm{k}$, and $\mathrm{q}_{\mathrm{j}}\left[\mathrm{s}_{\mathrm{j}-1}\right]=0$ (and $\mathrm{A}_{\mathrm{i}_{\mathrm{g}}}\left[\mathrm{t}_{j}\right]=0, \mathrm{~g}=1, \ldots, \mathrm{k}$, and $\left.\mathrm{Q}_{j}\left[\mathrm{t}_{\mathbf{j}}\right]=0\right), \mathrm{q}_{\mathbf{j}}\left[\mathrm{u}_{j}\right]$ is orthogonal to each $\mathrm{A}_{\mathrm{i}_{\mathrm{g}}}\left[\mathrm{u}_{\mathrm{j}}\right]$. However, as the intersection of a subspace and its orthogonal complement is zero, $Q_{j}\left[u_{j}\right]=0$, and the positions $u_{j}$ of any $q_{j} \in 2_{j}$ are zero.

We have thus identified three sets of row indices in $2_{j}$ that are zero for any full rank matrix $A \in \mathscr{b}$ with $A=Q R$. In particular, the presence of a Hall set in columns $\left\{\mathscr{t}_{1}, \ldots, \mathscr{C}_{j-1}\right\}$ means that $q_{i, j}=0$ for $i \in s_{j-1}$ (in 
fact, $q_{i, j+k}=0$ for $i \in s_{j-1}$ and $\left.k=0, \ldots, n-j\right)$. If $i \in t_{j}$, that is $a_{i k}=0$ for $k=1, \ldots, j$, then $q_{i j}=0$. If $i \in u_{j}$, then $q_{i j}=0$ because of combinatorial orthogonality in the columns of $\mathscr{G}$ which occurs in a subtle way (see corollary 4.9). 


\section{Strong Hall Bipartite Graphs.}

In this section we analyze the strong Hall property from a graph-theoretic point of view. The objective is to obtain a result (theorem $3.2)$ concerning the bipartite graphs $B_{j}(\not)$ defined in section 2 . We first prove a preliminary result concerning strong Hall bipartite graphs, which we now define.

Definition. Let $G(X, Y ; E)$ denote a bipartite graph with vertex sets $X, Y$ and undirected edge set $E$ and if $S \subseteq Y$, let $N_{G}(S)$ denote the set of all neighbors in $X$ of vertices in $S$. Then $G(X, Y ; E)$ is strong Hall with respect to $\mathrm{Y}$ if

(i) $|\mathrm{X}|=|\mathrm{Y}|>1$ and $|\mathrm{S}|<\left|\mathrm{N}_{\mathrm{G}}(\mathrm{S})\right|$, for all proper nonempty subsets $S$ of $Y$, or

(ii) $|\mathrm{X}|>|\mathrm{Y}|$ and $|\mathrm{S}|<\left|\mathrm{N}_{\mathrm{G}}(\mathrm{S})\right|$, for all nonempty subsets $\mathrm{S}$ of $\mathrm{Y}$.

There is an obvious equivalence between patterns $\mathscr{6}$ which satisfy the strong Hall property and bipartite graphs $G(X, Y ; E)$ that are strong Hall with respect to $\mathrm{Y}$ when the vertex sets $\mathrm{X}, \mathrm{Y}$ are associated with the row and column sets of $\mathscr{G}$, respectively, and edges correspond to nonzero entries in G. Note that the bipartite graphs $B_{j}(\mathscr{C})$ defined in section 2 are strong Hall with respect to $c_{j}(\hat{O}) \backslash\left\{c_{j}\right\}$.

The following theorem is used to prove theorem 3.2, but is of independent interest as it gives a characterization of strong $\underline{\text { Hall }}$ critical graphs $G=(X, Y ; E)$ with $|X|>|Y|$ (i.e., strong Hall graphs that are no longer strong Hall if any one edge is removed). Let $G$ be strong Hall with respect 
to $Y$. If for all $y \in Y$, the degree $d_{G}(y)=2$, then it is clear that $G$ is strong Hall critical with respect to $Y$. He now show that the converse is also true.

Theorem 3.1. Let $G=(X, Y ; E)$ be strong Hall with respect to $Y$ where $|\mathrm{X}|>|\mathrm{Y}|$. If $\mathrm{y} \in \mathrm{Y}$ has degree $\mathrm{d}_{G}(\mathrm{y}) \geq 3$, then there is at most one edge $\mathrm{e}=\{\mathrm{x}, \mathrm{y}\} \in \mathrm{E}$ such that $\mathrm{G}-\mathrm{e}$ is not strong Hall with respect to $\mathrm{Y}$.

Proof: Suppose there exists $y \in Y$ with $d_{G}(y) \geq 3$, and there is an edge $\mathrm{e}=\{\mathrm{x}, \mathrm{y}\} \in \mathrm{E}$ such that $\mathrm{H}=\mathrm{G}-\mathrm{e}$ is not strong Hall with respect to $\mathrm{Y}$. Let $S \subseteq Y, S \neq \emptyset$, be such that $\left|\mathrm{N}_{\mathrm{H}}(S)\right| \leq|S|$. If $\mathrm{y} \notin S$, then $N_{H}(S)=N_{G}(S)$ and hence $\left|N_{H}(S)\right|=\left|N_{G}(S)\right|>|S|$ since $G$ is strong Hall with respect to $Y$. Therefore $y \in S$. If $x \in N_{G}(S \backslash\{y\})$, then again $N_{H}(S)=N_{G}(S)$, a contradiction, and so $x \notin N_{G}(S \backslash\{y\})$. Thus $\left|N_{G}(S)\right|=$ $\left|N_{H}(S)\right|+1 \leq|S|+1$ and since $G$ is strong Hall with respect to $Y$, $\left|N_{G}(S)\right|=|S|+1$.

Now suppose there exists $y \in Y$ with $d_{G}(y) \geq 3$ and there exist distinct edges $e_{1}=\left\{x_{1}, y\right\}$ and $e_{2}=\left\{x_{2}, y\right\}$ in $E$ such that for $i=1,2$, $H_{i}=G-e_{i}$ is not strong Hall with respect to $Y$. Then for $i=1,2$ there exists $S_{i} \subseteq Y, S_{i} \neq \emptyset$, such that $\left|N_{H_{i}}\left(S_{i}\right)\right| \leq\left|S_{i}\right|$. From above, y $\in S_{1} \cap S_{2}$ and for $i=1,2$

$$
\left|N_{G}\left(S_{i}\right)\right|=\left|S_{i}\right|+1
$$

and

$$
x_{i} \notin N_{G}\left(S_{i} \backslash\{y\}\right)
$$

First suppose $\left(S_{1} \cap S_{2}\right) \backslash\{y\}=S \neq \emptyset$. For each $z \in S$ and $i=1,2$, $\left\{z, x_{i}\right\}$ is not an edge in $G$ by (3.2). Thus 


$$
\begin{aligned}
\left|N_{G}\left(S_{1} \cap S_{2}\right)\right| & =\left|N_{G}(\{y\}) \backslash N_{G}(S)\right|+\left|N_{G}(S)\right| \\
& \geq 2+\left|N_{G}(S)\right| \text { since } x_{1}, x_{2} \notin N_{G}(S) \\
& >2+|S| \text { since } S \neq \emptyset \text { and } G \text { is strong Hall } \\
& =2+\left(\left|S_{1} \cap S_{2}\right|-1\right) \\
& =\left|S_{1} \cap S_{2}\right|+1 .
\end{aligned}
$$

If, on the other hand, $S_{1} \cap S_{2}=\{y\}$, then

$$
\left|N_{G}\left(S_{1} \cap S_{2}\right)\right|=\left|N_{G}(\{y\})\right| \geq 3>\left|S_{1} \cap S_{2}\right|+1 .
$$

Therefore in any case

$$
\left|N_{G}\left(S_{1}\right) \cap N_{G}\left(S_{2}\right)\right| \geq\left|N_{G}\left(S_{1} \cap S_{2}\right)\right| \geq\left|S_{1} \cap S_{2}\right|+2 .
$$

\section{Thus}

$$
\begin{aligned}
& \left|S_{1} \cup S_{2}\right|=\left(\left|S_{1}\right|+1\right)+\left(\left|S_{2}\right|+1\right)-\left(\left|S_{1} \cap S_{2}\right|+2\right) \\
& \geq\left|N_{G}\left(S_{1}\right)\right|+\left|N_{G}\left(S_{2}\right)\right|-\left|N_{G}\left(S_{1}\right) \cap N_{G}\left(S_{2}\right)\right| \quad \text { by }(3.1) \text { and } \\
& =\left|N_{G}\left(S_{1}\right) \cup N_{G}\left(S_{2}\right)\right| \\
& =\left|N_{G}\left(S_{1} \cup S_{2}\right)\right| .
\end{aligned}
$$


This contradicts that $G$ is strong Hall with respect to $Y$ (note that $|\mathrm{X}|>|\mathrm{Y}|)$. Hence, at most one such edge $e_{i}$ can exist.

The next theorem provides a matching property of strong Hall bipartite graphs that is used to prove the main theorem in section 4 . (Recall that a matching II in a bipartite graph $G$ is a subset of its edges no two of which are adjacent. We say that II saturates a subset $S$ of the vertices of $G$ if every vertex in $S$ is incident with one of the edges in M.) He will use the following notation in the next theorem. If $\gamma_{1}$ and $\gamma_{2}$ are paths in a graph G, and $\gamma_{1}$ ends in a vertex adjacent in $G$ to the first vertex in $\gamma_{2}$, then $\gamma_{1} \gamma_{2}$ denotes the juxtaposition of $\gamma_{1}$ and $\gamma_{2}$ as alternating sequences of vertices and edges so that the edge from the end of $\gamma_{1}$ to the beginning of $\gamma_{2}$ is added giving a single path.

Theorem 3.2. Let $\mathrm{G}=(\mathrm{X}, \mathrm{Y} ; \mathrm{E})$ be a bipartite graph with $|\mathrm{X}| \geq|\mathrm{Y}|$, let $\mathrm{y} \in \mathrm{Y}$, and suppose that $\mathrm{G}-\mathrm{y}$ is strong Hall with respect to $\mathrm{Y} \backslash\{\mathrm{y}\}$. If there is a path $\gamma$ from $y$ to $x$ for some $x \in X$, then there exists a path $\tau$ from $y$ to $x$ and a matching $\mathbb{H}$ in $G-\tau$ such that $\mathbb{H}$ saturates all vertices in $\mathrm{Y}$ that are not on the path $\tau$.

Proof: Suppose the statement of the theorem is false and let $G=(X, Y ; E)$ be a counterexample with the fewest edges. Then there exists $x \in X$ and a path $\gamma$ from $y$ to $x$. Let $\hat{Y}=Y \backslash\{y\}$ and $\hat{G}=G-y$. Since $G$ is a counterexample there must exist a vertex $\hat{\mathrm{y}} \in \mathrm{Y}$ not on $\gamma$. If $\mathrm{d}_{\hat{G}}(\hat{y}) \geq 3$, then by theorem 3.1 , there exists $\hat{\mathrm{e}}=\{\hat{\mathrm{x}}, \hat{\mathrm{y}}\}$ such that if $H=G-\hat{e}$, then $H-y$ is strong Hall with respect to $\hat{Y}$. Now $\gamma$ is a path in H also, and since $G$ is a counterexample with the fewest edges, there 
exists some path $\tau$ from $\mathrm{y}$ to $\mathrm{x}$ and a matching $\mathbb{H}$ in $\mathrm{H}-\tau$ such that $\mathbb{M}$ saturates all the vertices in $\mathrm{Y}$ that are not on the path $\tau$. But this matching and path are also in $G$, a contradiction.

Therefore $\mathrm{d}_{\hat{\mathrm{G}}}(\hat{\mathrm{y}})=2$ (note that $\mathrm{d}_{\hat{G}}(\hat{\mathrm{y}})>1$ since $\hat{G}$ is strong Hall with respect to $\hat{y}$ ). Let $x_{1}$ and $x_{2}$ be the neighbors of $\hat{y}$ and let $H=(U, V ; F)$ be the graph obtained from $G$ by identifying $x_{1}$ with $x_{2}$ and by removing $\hat{\mathrm{y}}$. That is, $\mathrm{U}=\left(\mathrm{X} \backslash\left\{\mathrm{x}_{1}, \mathrm{x}_{2}\right\}\right) \cup\left\{\mathrm{x}^{*}\right\}$ where $\mathrm{x}^{*} \notin \mathrm{X} \cup \mathrm{Y}$ and $\mathrm{V}=\mathrm{Y} \backslash\{\hat{\mathrm{y}}\}$. Moreover, for all $\mathrm{u} \in \mathrm{U}$ and $\mathrm{v} \in \mathrm{V},\{\mathrm{u}, \mathrm{v}\} \in \mathrm{F}$ if and only if

(i) $u \in X \backslash\left\{x_{1}, x_{2}\right\}, v \in V$ and $\{u, v\} \in E$; or

(ii) $u=x^{*}, v \in V$, and $\left\{x_{1}, v\right\} \in E$ or $\left\{x_{2}, v\right\} \in E$.

Let $\hat{\mathrm{V}}=\mathrm{V} \backslash\{\mathrm{y}\}, \hat{\mathrm{H}}=\mathrm{H}-\mathrm{y}$ and note that since $|\mathrm{X}|>|\hat{\mathrm{Y}}|$, we have $|\mathrm{U}|>|\hat{\mathrm{V}}|$.

Claim: $\hat{\mathrm{H}}$ is strong Hall with respect to $\hat{\mathrm{V}}$.

Proof of Claim: Let $S \subseteq \hat{V}, S \neq \emptyset$. If $x^{*} \notin N_{\hat{H}}(S)$, then $x_{1} \notin N_{\hat{G}}(S)$ and $\mathrm{x}_{2} \notin \mathrm{N}_{\hat{\mathrm{G}}}(\mathrm{S})$. Thus $\mathrm{N}_{\hat{\mathrm{H}}}(\mathrm{S})=\mathrm{N}_{\hat{\mathrm{G}}}(\mathrm{S})$ and since $\hat{\mathrm{G}}$ is strong Hall with respect to $\hat{\mathrm{Y}},|\mathrm{S}|<\left|\mathrm{N}_{\hat{\mathrm{G}}}(\mathrm{S})\right|=\left|\mathrm{N}_{\hat{\mathrm{H}}}(\mathrm{S})\right|$.

Suppose there exists $S \subseteq \hat{V}$ such that $|S| \geq\left|N_{\hat{H}}(S)\right|$. Then $x^{*} \in N_{\hat{H}}(S)$, by the above, and hence at least one of $x_{1}$ or $x_{2}$ is contained in $N_{\hat{G}}(S)$. If only one is contained in $N_{\hat{G}}(S)$, then since it is only replaced with $x^{*}$ in $H$, we have $\left|N_{\hat{G}}(S)\right|=\left|N_{\hat{H}}(S)\right|$, a contradiction to $|S|<\left|N_{\hat{G}}(S)\right|$. Thus both $\mathrm{x}_{1}$ and $\mathrm{x}_{2}$ are contained in $\mathrm{N}_{\hat{G}}(\mathrm{~S})$. Since $\mathrm{x}_{1}$ and $\mathrm{x}_{2}$ are replaced with $x^{*}$ in $H,\left|N_{\hat{G}}(S)\right|=\left|N_{\hat{H}}(S)\right|+1$ and $N_{\hat{G}}(S U\{\hat{y}\})=N_{\hat{G}}(S)$. But then 


$$
|S \cup\{\hat{y}\}|=|S|+1 \geq\left|N_{\hat{H}}(S)\right|+1=\left|N_{\hat{G}}(S)\right|=\left|N_{\hat{G}}(S U\{\hat{y}\})\right|
$$

contradicts the fact that $\hat{G}$ is strong Hall with respect to $\hat{\mathrm{Y}}$ (note that $\mathrm{S} U\{\hat{\mathrm{y}}\} \subseteq \hat{\mathrm{Y}})$. This completes the proof of the claim.

We now identify a path, $\eta$ in $H$ that is dependent upon the path $\gamma$ in G. The following table describes how $\eta$ is defined in $H$ given the structure of $\gamma$. In the table, $\pi, \rho$ and $\sigma$ are nonempty paths (i.e., they each contain at least one vertex).

\begin{tabular}{|l|l|l|}
\hline Case & $\gamma$ is & path $\eta$ in $\mathrm{H}$ is \\
\hline
\end{tabular}

\begin{tabular}{|c|c|c|}
\hline 1 & $\gamma\left(\mathrm{x}_{1}\right.$ and $\mathrm{x}_{2}$ not on $\left.\gamma\right)$ & $\gamma$ \\
\hline 2 & $\pi \mathrm{x}_{1} \rho$ or $\pi \mathrm{x}_{2} \rho$ & $\pi \mathrm{x}^{*} \rho$ \\
\hline 3 & $\pi \mathrm{x}_{1} \rho \mathrm{x}_{2} \sigma$ or $\pi \mathrm{x}_{2} \rho \mathrm{x}_{1} \sigma$ & $\pi \mathrm{x}^{*} \sigma$ \\
\hline 4 & $\pi \mathrm{x}_{1} \rho \mathrm{x}_{2}$ or $\pi \mathrm{x}_{2} \rho \mathrm{x}_{1}$ & $\pi \mathrm{x}^{*}$ \\
\hline 5 & $\pi \mathrm{x}_{1}$ or $\pi \mathrm{x}_{2}$ & $\pi \mathrm{x}^{*}$ \\
\hline
\end{tabular}

Let $\eta$ end at vertex $u$. Note that $u \in \mathbb{U}$ and that $\eta$ starts at vertex $y$. Since $\hat{H}$ is strong Hall with respect to $\hat{V}$, and $H$ has fewer edges than $G$, there exists a path $\omega$ in $H$ from $y$ to $u$ and matching $\hat{\mathbb{H}}$ in $\mathrm{H}-\omega$ which saturates all vertices in $\mathrm{V}$ that are not on $\omega$. He will use this path and matching to construct similar ones in $G$. The constructions will differ depending on the structure of $\gamma$ in $G$, but each will contradict 
that $G$ is a counterexample to the theorem . The cases below are described in the table above.

Cases 1-3. Here we have $x_{1} \neq u \neq x_{2}$ and hence $u=x$.

If $x^{*}$ is not on $\omega$ and $x^{*}$ is not saturated by $\hat{M}$, then $\omega$ is a path in $G$ from $y$ to $x$ and $\mathbb{M}=\hat{\mathbb{H}} \cup\left\{\left\{x_{1}, \hat{y}\right\}\right\}$ is a matching in $G$ which saturates all vertices in $\mathrm{Y}$ that are not on $w$, a contradiction.

If there exists a $v \in V$ such that $\left\{x^{*}, v\right\} \in \hat{\mathbb{M}}$, then $x^{*}$ is not on $\omega$ and hence $\omega$ is a path in $G$ from $y$ to $x$. Moreover, $\{z, v\} \in E$ for some $z \in\left\{x_{1}, x_{2}\right\}$. Let $\{w\}=\left\{x_{1}, x_{2}\right\} \backslash\{z\}$. Thus $M=\left(\hat{\mathbb{M}} \backslash\left\{\left\{\mathrm{x}^{*}, \mathrm{v}\right\}\right\}\right) \cup\{\{\mathrm{z}, \mathrm{v}\},\{\mathrm{w}, \hat{\mathrm{y}}\}\}$ is a matching in $\mathrm{G}$ which saturates all vertices in $\mathrm{Y}$ that are not on $\omega$, a contradiction.

Therefore $x^{*}$ is on $\omega$ and $\hat{H}$ is a matching in G. Let $\omega=\omega_{1} x^{*} \omega_{2}$ and let $v_{1}$ and $v_{2}$ be the neighbors of $x^{*}$ on $\omega$ such that $w_{1}$ ends at $v_{1}$ and $w_{2}$ starts at $v_{2}$. If $\left\{\mathrm{x}_{1}, \mathrm{x}_{2}\right\} \subseteq \mathrm{N}_{\mathrm{G}}\left(\left\{\mathrm{v}_{1}, \mathrm{v}_{2}\right\}\right)$, then at least one of $\tau_{1}=\omega_{1} \mathrm{x}_{1} \hat{\mathrm{yx}} \mathrm{x}_{2} \omega_{2}$ and $\tau_{2}=\omega_{1} \mathrm{x}_{2} \hat{\mathrm{yx}} \omega_{1} \omega_{2}$ is a path in $G$ that starts at $y$ and ends at $x$. Thus for some $i \in\{1,2\}, \hat{\mathbb{M}}$ saturates all vertices in $\mathrm{Y}$ that are not on $\tau_{i}$, a contradiction. Hence $\left\{x_{1}, x_{2}\right\} \nsubseteq N_{G}\left(\left\{v_{1}, v_{2}\right\}\right)$. Thus since $x^{*}$ is adjacent to $v_{1}$ and $\mathrm{v}_{2}$, they are both adjacent to $\mathrm{x}_{1}$ or to $\mathrm{x}_{2}$ in $G$. If $\mathrm{v}_{1}$ and $\mathrm{v}_{2}$ are neighbors of $x_{1}$, then $\tau=\omega_{1} x_{1} w_{2}$ is a path in $G$ from $y$ to $x$, and $y=\hat{H} \cup\left\{\left\{x_{2}, \hat{y}\right\}\right\}$ is a matching in $G$ that saturates all vertices in $Y$ that are not on $\tau$, a contradiction. Similarly, if $v_{1}$ and $v_{2}$ are neighbors of $x_{2}$, then $\tau=\omega_{1} x_{2} \omega_{2}$ is a path in $G$ from $y$ to $x$, and $\mathbb{M}=\hat{\mathbb{M}} \cup\left\{\left\{\mathrm{x}_{1}, \hat{\mathrm{y}}\right\}\right\}$ is a matching in $G$ that saturates all vertices in $\mathrm{Y}$ that are not on $\tau$, another contradiction. Therefore, Cases 1-3 cannot occur. 
Cases 4 and 5. Note that $u=x^{*}$ and hence $\omega$ ends at $x^{*}$. Moreover, $\hat{M}$ is a matching in G. Let $\omega=\omega_{1} x^{*}$ and let $v_{1}$ be the last vertex of the path $w_{1}$. Since $v_{1}$ is adjacent to $x^{*}$ in $H$, it is adjacent to either $\mathrm{x}_{1}$ or $\mathrm{x}_{2}$ in $G$. Thus either $\tau_{1}=w_{1} \mathrm{x}_{1}, \tau_{2}=w_{1} \mathrm{x}_{2} \hat{\mathrm{y}} \mathrm{x}_{1}$, $\tau_{3}=w_{1} x_{2}$, or $\tau_{4}=w_{1} x_{1} \hat{y} x_{2}$ is a path from $y$ to $x$ in $G$. If $\tau_{2}$ is such a path in $G$, then $\hat{\mathbb{M}}$ saturates all vertices in $Y$ that are not on $\tau_{2}$; similarly if $\tau_{4}$ is a path from $\mathrm{y}$ to $\mathrm{x}$ in G. Otherwise, $\hat{\mathbb{I}} \cup\left\{\left\{\mathrm{x}_{2}, \hat{\mathrm{y}}\right\}\right\}$ saturates all vertices in $\mathrm{Y}$ that are not on $\tau_{1}$ or $\hat{\mathbb{M}} \cup\left\{\left\{\mathrm{x}_{1}, \hat{\mathrm{y}}\right\}\right\}$ saturates all vertices in $\mathrm{Y}$ that are not on $\tau_{3}$, a contradiction. Therefore Cases 4 and 5 cannot occur.

Since these cases exhaust all possibilities, we have a contradiction. Therefore no such counterexample $G$ can exist and the theorem is proved. 
4. Nonzero entries in 2.

In section 2 , we have identified positions in 2 that must be zero for a variety of more or less subtle reasons. To complete the solution to our problem and see that we have identified all the necessarily zero positions in 2, we must show that for each remaining position there is a full rank matrix $A \in \mathscr{b}$ whose $Q R$ factorization has a nonzero entry in $Q$ in the position of interest. Our strategy is, for each nonzero position, to identify a particular full rank matrix A which produces a $Q$ with a nonzero in that position and whose pattern is contained in that of $\mathscr{G}$. A simple perturbation argument then yields an $A$ with the exact pattern of $\mathscr{b}$. We note that our argument is sufficient to prove that the zero/nonzero structure for 2 is tight (i.e., the best possible).

We first consider a particularly simple form for to. To describe our construction, we introduce the notation $\mathbf{Q}^{\mathrm{u}}$ for a matrix that has orthogonal columns that are not in general of unit length (i.e. unnormalized); $q_{j}^{\mathrm{u}}$ denotes the jth column of this matrix.

Lemma 4.1. Let $\mathscr{t}$ be an $\mathrm{f}$-by-f pattern with bipartite graph

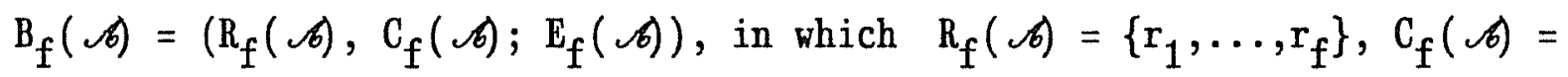
$\left\{c_{1}, \ldots, c_{f}\right\}$ and the edges in $E_{f}(\mathscr{f})$ form a simple path of length $2 f-1$ from $c_{f}$ to some $r_{i}, 1 \leq i \leq f$. There exists a nonsingular matrix $A \in \mathscr{b}$ such that if $\AA=Q R$, then $q_{\text {if }}$ is nonzero.

Proof: Assume that the path from $c_{f}$ to $r_{i}$ is 


$$
c_{f}=c_{j_{f}}, r_{k_{f}}, c_{j_{f-1}}, \ldots, c_{j_{2}}, r_{k_{2}}, c_{j_{1}}, r_{k_{1}}=r_{i} .
$$

Let $A=\left[a_{d e}\right]$ be the $f-b y-f(0,1)$ matrix with $a_{d e}=1$ iff $\left\{r_{d}, c_{e}\right\}$ lies on the path; thus $A \in \mathscr{G}$. Matrix $A$ has exactly 2 entries equal to 1 in each column (except the last). Since A is permutation equivalent to a lower triangular matrix with ones on the main diagonal, $\mathrm{A}$ is nonsingular. The columns (except $c_{f}$ ) can occur in any order on the path, but the exact order is important to the following construction of $q_{\mathrm{f}}^{\mathrm{u}}$.

Consider

$$
q_{f}^{u}=q_{j_{f}}^{u}=f A_{j_{f}}-(f-1) A_{j_{f-1}}+\ldots \neq 2 A_{j_{2}} \pm A_{j_{1}},
$$

where the columns are from matrix $A$, the multiplying factors have alternating signs and $f$ is the number of columns on the path. Clearly this $q_{j_{f}}^{u} \in \operatorname{span}\left\{A_{j_{1}}, \ldots, A_{j_{f}}\right\}$, and we need to check the orthogonality conditions. By the construction of $A$, we have $A_{j_{1}} \cdot A_{j_{1}}=2, A_{j_{1}} \cdot A_{j_{2}}=1$ and $A_{j_{1}} \cdot A_{j_{k}}=0$ for $k=3, \ldots, f$. Thus $q_{f}^{u} \cdot A_{j_{1}}=0$. Similarly $A_{j_{k}} \cdot A_{j_{k}}=2$ for $k=2, \ldots, f-1$, and $A_{j_{k}} \cdot A_{j_{k-1}}=A_{j_{k}} \cdot A_{j_{k+1}}=1$ for $k=2, \ldots, f-1$, with $A_{j_{k}}$ (combinatorially) orthogonal to all other columns. Thus $a_{f}^{u} \cdot A_{j_{k}}=0$ for $k=1, \ldots, f-1$. The construction of $A$ and the distinct multipliers in (4.1) mean that there can be no numerical cancellation, and so the ith entry of $Q_{f}^{u}$ is nonzero. It is clear that if the column $Q_{f}^{u}$ is normalized to have unit length, then column $f$ of the matrix $Q$ of the $Q R$ factorization of $A$ is obtained, with $q_{i f} \neq 0$. 
Note that the construction in lemma 4.1 guarantees that all entries in column $f$ of $Q$ are nonzero, not just $q_{\text {if }}$. To illustrate lemma 4.1, consider an example in which $i=2$ and $f=3$.

Example (4.2). Consider the pattern $\mathscr{b}$ and bipartite graph $\mathrm{B}_{3}(\mathscr{C})$ in Figure 2.

$$
\mathscr{6}=\left[\begin{array}{lll}
* & * & 0 \\
0 & * & 0 \\
* & 0 & *
\end{array}\right] \text { with } \mathrm{B}_{3}(\hat{\theta}):
$$

Figure 2.

The edges in $E_{3}(\mathscr{6})$ form one path from column $c_{3}$ to $r_{2}$, namely $c_{3}, r_{3}$, $c_{1}, r_{1}, c_{2}, r_{2}$. Take the matrix

$$
A=\left[\begin{array}{lll}
1 & 1 & 0 \\
0 & 1 & 0 \\
1 & 0 & 1
\end{array}\right] \in \mathscr{6}
$$

From (4.1), $\mathrm{Q}_{3}^{\mathrm{u}}=3 \mathrm{~A}_{3}-2 \mathrm{~A}_{1}+\mathrm{A}_{2}=(-1,1,1)^{\mathrm{T}}$, (note the order of the $\mathrm{A}_{\mathrm{i}}$ in this equation is determined by the path), and the $(2,3)$ entry of $Q^{\mathrm{u}}$ is equal to 1 . Thus if $A=Q R$, column 3 of $Q$ must be a scalar multiple of $Q_{3}^{u}$ (by the uniqueness of the $Q R$ factorization), and in fact 


$$
Q=\left[\begin{array}{rrr}
\frac{1}{\sqrt{2}} & \frac{1}{\sqrt{6}} & -\frac{1}{\sqrt{3}} \\
0 & \frac{2}{\sqrt{6}} & \frac{1}{\sqrt{3}} \\
\frac{1}{\sqrt{2}} & -\frac{1}{\sqrt{6}} & \frac{1}{\sqrt{3}}
\end{array}\right] .
$$

Example (4.3). In the event that $\mathscr{C}$ has bipartite graph $\mathrm{B}_{\mathrm{f}}(\mathscr{C})$ with edges forming a path traversing the columns in order $c_{f}, c_{f-1}, \ldots, c_{1}$, then $A \in \mathscr{C}$ is bidiagonal. For example when $f=5$,

$$
A=\left[\begin{array}{lllll}
1 & 0 & 0 & 0 & 0 \\
1 & 1 & 0 & 0 & 0 \\
0 & 1 & 1 & 0 & 0 \\
0 & 0 & 1 & 1 & 0 \\
0 & 0 & 0 & 1 & 1
\end{array}\right] \in \mathscr{C}
$$

the corresponding $Q^{u}$ is the full upper Hessenberg matrix

$$
\mathbf{Q}^{\mathrm{u}}=\left[\begin{array}{rrrrr}
1 & -1 & 1 & -1 & 1 \\
1 & 1 & -1 & 1 & -1 \\
0 & 2 & 1 & -1 & 1 \\
0 & 0 & 3 & 1 & -1 \\
0 & 0 & 0 & 4 & 1
\end{array}\right]
$$

and note that $R$ is bidiagonal upper triangular.

To prove our result for more general patterns, we need the fact that $Q$ depend̄s continuously on $\AA$. Hore specificaliy, if $\AA$ is a matrix with fuli column rank and $A=Q R$, then there exists a neighborhood of $A$ such that every matrix in this neighborhood has full column rank and the orthogonal matrix of its factorization depends continuously on the entries. This qualitative fact (which is all we need) may be straightforwardly proven by 
elementary means, but precise bounds have been given in [S, Theorem 3.1] . He now use continuity to prove our main result on nonzero entries in 2.

Theorem 4.4. For $\mathrm{m} \geq \mathrm{n}$, let $\mathscr{6}$ be an $\mathrm{m}$-by-n pattern with the Hall property. If $r_{i} \in p_{j}, 1 \leq j \leq n$, then there is an $A \in \mathscr{C}$ of rank $n$ such that $A=Q R$ with $q_{i j}$ nonzero.

Proof: Without loss of generality, we can assume that each diagonal entry of $\mathscr{G}$ is *. (Thus, as noted in section $2, \mathrm{~s}_{j}=\left\{\mathrm{i}: 1 \leq \mathrm{i} \leq \mathrm{j}\right.$ and $\left.\mathscr{C}_{\mathrm{i}} \in \mathrm{s}_{\mathrm{j}}\right\}$ and consequently if some column $c_{\mathrm{e}} \notin \mathrm{C}_{\mathrm{j}}(\mathscr{\sigma})$, where $1 \leq \mathrm{c}_{\mathrm{e}}<\mathrm{j}$, then $\left.\mathrm{r}_{\mathrm{e}} \notin \mathrm{R}_{\mathrm{j}}(\mathscr{\mathscr { C }}).\right)$ As $\mathrm{r}_{\mathrm{i}} \in \mathrm{p}_{\mathrm{j}}$, there exists at least one path in $\mathrm{B}_{\mathrm{j}}(\mathscr{\mathscr { C }})$ from $c_{j}$ to $r_{i}$. Thus, by Theorem 3.2 , there exists a path, say

$$
c_{j}=c_{j_{f}}, r_{k_{f}}, c_{j_{f-1}}, \ldots, c_{j_{2}}, r_{k_{2}}, c_{j_{1}}, r_{k_{1}}=r_{i}
$$

from $c_{j}$ to $r_{i}$ of length $2 f-1 \quad(1 \leq f \leq j)$ and a matching $M$ in $B_{j}(\mathscr{b})$ of all columns in $B_{j}(\mathscr{B})$ that are not on the path (4.2) to some subset of the rows in $B_{j}(\mathscr{G})$ that are not on the path (4.2). For any nonzero $\epsilon$, let $A_{\epsilon}=\left[a_{\mathrm{de}}\right]$ be the $\mathrm{m}-\mathrm{by}-\mathrm{j}$ matrix whose pattern $\mathscr{\mathscr { t }}_{\epsilon}$ is the same as the first $\mathrm{j}$ columns of $\mathscr{L}$ and such that $\mathrm{a}_{\mathrm{de}} \in\{0,1, \epsilon\}$ with nonzero terms given by the following construction: 


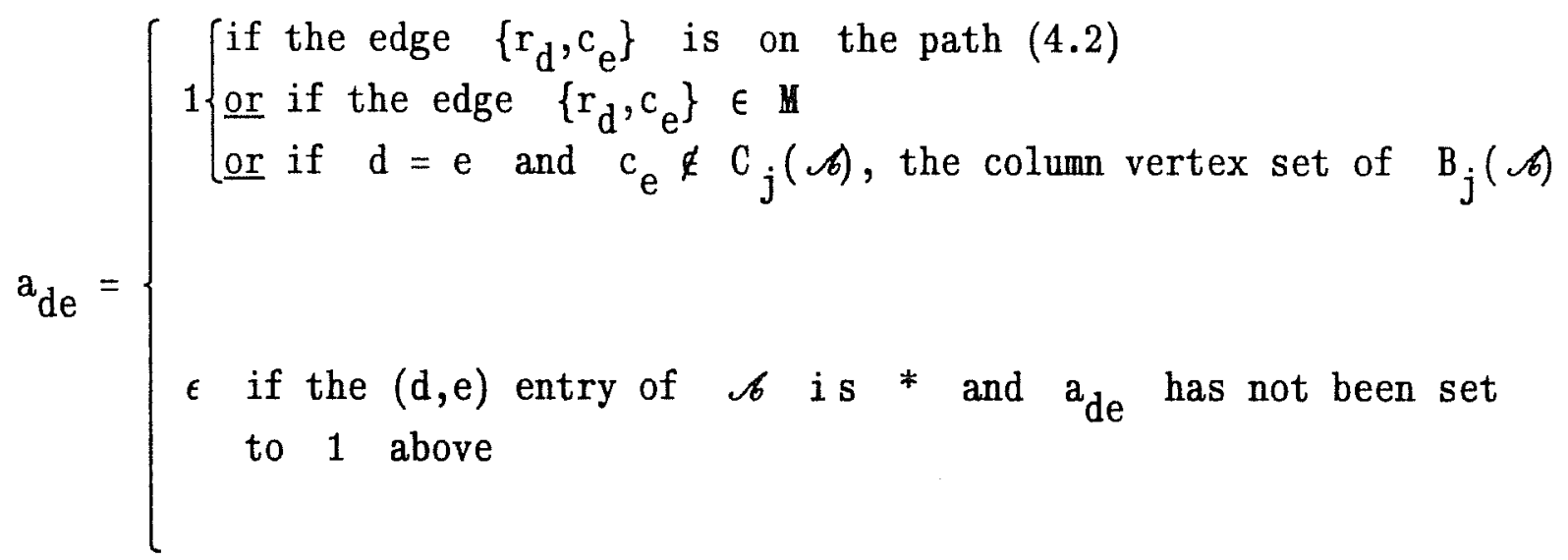

(Note that $\mathscr{c}_{\mathrm{j}+1}, \ldots, \mathscr{c}_{\mathrm{n}}$ do not enter here.)

Let $A_{0}$ denote the matrix $A_{\epsilon}$ with all $\epsilon$ entries set to zero, and let $\tilde{A}=\left[\tilde{a}_{\mathrm{de}}\right]$ be the $(0,1)$ square submatrix of $A_{0}$ restricted to rows $r_{k_{1}}, \ldots, r_{k_{f}}$ and columns $c_{j_{1}}, \ldots, c_{j_{f}}$, with $\tilde{a}_{d e}=1$ if and only if $\mathrm{a}_{\mathrm{de}}=1$. Let $\tilde{\mathscr{G}}$ be the pattern of $\tilde{A}$; then $\tilde{\mathscr{H}}_{\boldsymbol{G}}$ satisfies the conditions of lemma 4.1 and $\tilde{A}$ is the matrix of the construction in lemma 4.1. Thus if $\tilde{A}=\tilde{Q} \tilde{R}$, then $\tilde{q}_{i j}$ is nonzero.

Now we consider the matrix $A_{0}$, and note that $\tilde{A}$ is embedded in $A_{0}$. By construction, columns of $A_{0}$ not on the path (4.2) contain distinct unit vectors, so $A_{0}$ has full column rank. If $A_{0}=Q_{0} R_{0}$, then $\tilde{Q}$ is embedded in $Q_{0}$ in the same way as $\tilde{A}$ is embedded in $A_{0}$, with corresponding unit column vectors in columns not on the path. Thus the $(i, j)$ entry of $Q_{0}$ is equal to $\tilde{\mathrm{q}}_{\mathrm{ij}}$ and is nonzero.

Finally, consider $A_{\epsilon}=Q_{\epsilon} R_{\bar{\epsilon}}$, which has the same column rank as $A_{0}$, namely $j$, for sufficiently small $\epsilon$. By the continuity statement above, for sufficiently small $\epsilon$, the matrix $Q_{\epsilon}$ has its $(i, j)$ entry nonzero. Thus any full rank matrix $A \in \mathscr{C}$ with the submatrix in its first $j$ columns equal to $A_{\epsilon}$ has $q_{i j}$ nonzero, where $A=Q R$, since column $j$ of $Q$ is independent of $A_{j+1}, \ldots, A_{n}$. 
To illustrate this construction, consider an example.

Example (4.5). Consider the pattern $\mathscr{b}$ and bipartite graph $\mathrm{B}_{4}(\mathscr{\mathscr { C }})$ in Figure 3 .

$$
\mathscr{6}=\left[\begin{array}{lllll}
* & * & * & 0 & 0 \\
0 & * & 0 & 0 & 0 \\
0 & 0 & * & 0 & * \\
* & 0 & 0 & * & 0 \\
0 & 0 & * & 0 & * \\
0 & * & 0 & 0 & 0
\end{array}\right] \text { with } \mathrm{B}_{4}(\mathscr{C}):
$$

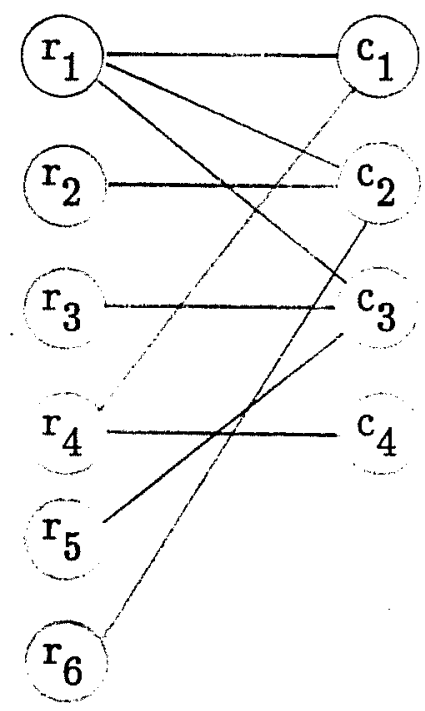

Figure 3.

There is one path in $B_{4}(\sigma)$ from $c_{4}$ to $r_{2}$, namely $c_{4}, r_{4}, c_{1}, r_{1}$, $\mathrm{c}_{2}, \mathrm{r}_{2}$, implying that the $(2,4)$ entry of 2 is *. As this is the only path in $\mathrm{B}_{4}(\mathscr{G})$ from $\mathrm{c}_{4}$ to $\mathrm{r}_{2}$, this must be the path (4.2) in the proof of theorem 4.4, and the matching $M$ can be chosen as either the edge $\left\{\mathrm{r}_{3}, \mathrm{c}_{3}\right\}$ or the edge $\left\{r_{5}, c_{3}\right\}$. If the first of these, then

$$
A_{\epsilon}=\left[\begin{array}{llll}
1 & 1 & \epsilon & 0 \\
0 & 1 & 0 & 0 \\
0 & 0 & 1 & 0 \\
1 & 0 & 0 & 1 \\
0 & 0 & \epsilon & 0 \\
0 & \epsilon & 0 & 0
\end{array}\right] \quad \text { and } \tilde{\AA}=\left[\begin{array}{lll}
1 & 1 & 0 \\
0 & 1 & 0 \\
1 & 0 & 1
\end{array}\right]
$$

From the proof of lemma 4.1 , the third column of $\tilde{Q}$ is a scalar multiple of 
$(-1,1,1)^{\mathrm{T}}$, implying that the fourth column of $Q_{0}$ is a scalar multiple of $(-1,1,0,1,0,0)^{\mathrm{T}}$. Thus, by continuity, the $(2,4)$ entry of $q_{\epsilon}$ is nonzero, implying that the $(2,4)$ entry of 2 is nonzero.

Consider now a further example, with a pattern having more than one path from $c_{j}$ to $r_{i}$ (cf. example (4.5) with a change in column 1). Example (4.6). Consider the pattern $\mathscr{6}$ and bipartite graph $\mathrm{B}_{3}(\mathscr{b})$ in Figure 4.

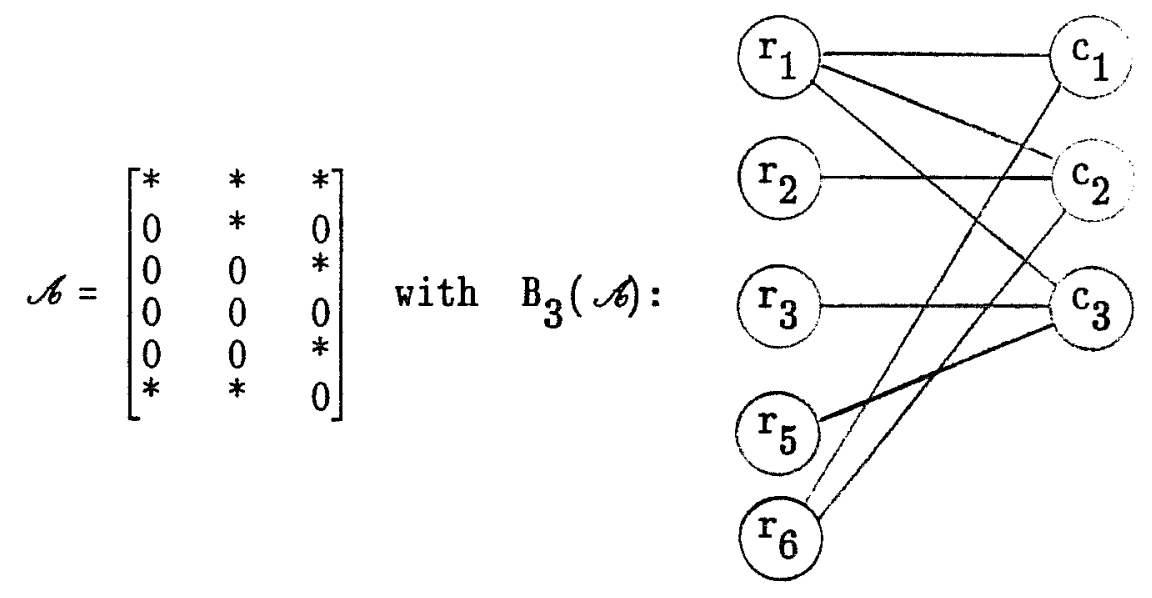

Figure 4 .

Focussing on the $(6,3)$ entry, there are 2 paths, namely $c_{3}, r_{1}, c_{1}, r_{6}$ and $c_{3}, r_{1}, c_{2}, r_{6}$, from $c_{3}$ to $r_{6}$. The path (4.2) in the proof of theorem 4.4 (i.e., the path $\tau$ in theorem 3.2) is the first of these, and the matching $I I$ is the edge $\left\{r_{2}, c_{2}\right\}$. Note that for the other path from $c_{3}$ to $\mathrm{r}_{6}$, there is no matching in the bipartite graph of $c_{1}$ with any row vertex not on this path (i.e., $r_{2}, r_{3}, r_{4}$ or $r_{5}$ ). This illustrates the care required in the selection of a path (4.2) in the proof of theorem 4.4; there must exist an associated matching $\mathbb{H}$ so that $A_{\epsilon}$ is constructed such that $A_{0}$ has full 
column rank. The result of theorem 3.2 ensures that this is always possible. With the choice of the path $c_{3}, r_{1}, c_{1}, r_{6}$ above, we obtain

$$
A_{\epsilon}=\left[\begin{array}{lll}
1 & \epsilon & 1 \\
0 & 1 & 0 \\
0 & 0 & \epsilon \\
0 & 0 & 0 \\
0 & 0 & \epsilon \\
1 & \epsilon & 0
\end{array}\right] \epsilon \mathscr{6}
$$

For sufficiently small $\epsilon$, if $A_{\epsilon}=A=Q R$, then $q_{63}$ is nonzero.

We now combine the results of theorems 2.1 and 4.4 to solve the problem stated in the introduction (as far as 2 is concerned) in terms of the row index partition defined in section 2 .

Theorem 4.7. For $\mathrm{m} \geq \mathrm{n}$, let $\mathscr{b}$ be an $\mathrm{m}-\mathrm{by}-\mathrm{n}$ pattern with the Hall property. Then the $(i, j)$ entry of 2 is zero if and only if $i \in s_{j-1} \cup t_{j} \cup u_{j}$. Equivalently, the $(i, j)$ entry of $\mathcal{L}$ is nonzero if and only if $i \in p_{j}$.

The next two corollaries indicate how 2 can be computed. In the strong Hall case, $s_{j-1}=\emptyset, 2 \leq j \leq n$, and $\mathscr{q}_{j}$ is the union of $\mathscr{C}_{j}$ and certain columns $\mathscr{G}_{\mathrm{W}}, 1 \leq \mathrm{w} \leq \mathrm{j}-1$.

Corollary 4.8. For $\mathrm{m} \geq \mathrm{n}$, let $\mathscr{6}$ be an $\mathrm{m}-$ by- $\mathrm{n}$ pattern with the strong Hall property. For any fixed $\mathrm{j}, 1 \leq \mathrm{j} \leq \mathrm{n}$, partition $\mathscr{G}_{1}, \ldots, \mathscr{G}_{\mathrm{j}}$ into two disjoint sets $\mathscr{b}_{j}$ and $\mathscr{Y}_{j}$, for which $\mathscr{b}_{j} \in \mathscr{o}_{j}$, every column of $\mathscr{b}_{j}$ is combinatorially orthogonal to every column of $y_{j}$, and $\left|y_{j}\right|$ is as large as possible. Then $\mathscr{2}_{\mathrm{j}}=U \mathscr{C}_{\mathrm{V}}, \mathscr{L}_{\mathrm{v}} \in \mathscr{O}_{\mathrm{j}}$. 
Proof: Since $S_{j-1}=\phi, 2 \leq j \leq n$, the bipartite graph $B_{j}(\mathscr{H})$ has $c_{j}(\mathscr{O})=\left\{c_{1}, \ldots, c_{j}\right\}$ and $R_{j}(\mathscr{O})=\left\{r_{i}=i\right.$ : there exists a $*$ in row $i$ in one of the columns of $\left.c_{j}(\mathscr{C})\right\}$. For $k \leq j$, column $c_{k} \in \mathscr{O}_{j}$ if and only if there exists a path from $c_{j}$ to some $r_{i}$ where column $c_{k}$ has a $*$ in row $r_{i}$. By definition of $p_{j}$, this is true if and only if $r_{i} \in p_{j}$; and by theorem 4.7, $r_{i} \in p_{j}$ if and only if $q_{i j}$ is nonzero. Together these give exactly the union statement above.

For th having the Hall property, the essential modification to the result of the above corollary is that in the union of columns $\mathscr{G}_{\mathrm{W}}$ specifying $\mathscr{2}_{\mathrm{j}}$, the column vectors in $S_{j-1}$ and nonzeros in rows $s_{j-1}$ must be omitted.

Corollary 4.9. For $\mathrm{m} \geq \mathrm{n}$, let $\mathscr{6}$ be an $\mathrm{m}-$ by-n pattern with the Hall property. For any fixed $j$, consider the subpattern of $\mathscr{b}$ in columns $\left\{\mathscr{c}_{1}, \ldots, \mathscr{c}_{\mathrm{j}}\right\}-\mathrm{s}_{\mathrm{j}-1}$ and rows $\mathrm{v}_{\mathrm{j}}=\{1, \ldots, \mathrm{m}\}-\mathrm{s}_{\mathrm{j}-1}$; this is an $\left(m-\left|s_{j-1}\right|\right)-b y-\left(j-\left|s_{j-1}\right|\right)$ pattern. Partition the columns of this subpattern into 2 disjoint sets, $\mathscr{b}_{j}$ and $\mathscr{H}_{j}$, with $\mathscr{b}_{j}\left[\mathrm{v}_{j}\right] \in \mathscr{b}_{j}$, every column of $\mathscr{B}_{j}$ is combinatorially orthogonal to every column of $y_{j}$ and $\left|y_{j}\right|$ is as large as possible. Then $\mathscr{2}_{\mathrm{j}}\left[\mathrm{s}_{\mathrm{j}-1}\right]=0$ and $\mathscr{2}_{\mathrm{j}}\left[\mathrm{v}_{\mathrm{j}}\right]=U \mathscr{L}_{\mathrm{w}}\left[\mathrm{v}_{\mathrm{j}}\right]$, with $\mathscr{C}_{\mathrm{W}}\left[\mathrm{v}_{\mathrm{j}}\right] \in \mathscr{O}_{\mathrm{j}}$.

Proof: By definition, $p_{j}$ is the set of ail row indices $r_{i}$ such that

$$
\mathrm{r}_{\mathrm{i}} \notin \mathrm{s}_{\mathrm{j}-1}
$$

(ii) there exists a column $c_{k}, 1 \leq k \leq j$, such that $\mathscr{k}_{k} \notin \mathrm{S}_{\mathrm{j}-1}$ and there is $a *$ in row $r_{i}$ of $c_{k}$ and (iii) there exists a path in $B_{j}(\infty)$ from $c_{j}$ to $r_{i}$. 
Thus the positions of nonzeros in vectors $\mathscr{b}_{\mathrm{W}}\left[\mathrm{v}_{\mathrm{j}}\right] \in \mathscr{O}_{\mathrm{j}}$ correspond precisely to entries in $p_{j}$, and the result follows from theorem 4.7 .

The following algorithm implements the construction in corollary 4.9.

Algorithm 4.10. Computation of the Pattern 2.

Notation:

$\mathscr{6}$ is an $\mathrm{m}-\mathrm{by}-\mathrm{n}$ pattern, $\mathrm{m} \geq \mathrm{n}$, satisfying the Hall property.

$\mathscr{C}_{\mathrm{j}}$ is the $\mathrm{j}$-th column of $\mathscr{C}, 1 \leq \mathrm{j} \leq \mathrm{n}$.

$\beta\left(\mathscr{C}_{\mathrm{j}}\right)=\left\{\mathrm{i} \mid\right.$ the $\mathrm{i}$-th entry of $\mathscr{C}_{\mathrm{j}}$ is $\left.{ }^{*}\right\}$.

$S_{j}, s_{j}$ are as defined in section 2 .

1. For $1 \leq j \leq n-1$, compute $S_{j}$ and $s_{j}$.

2. Initialization

$$
\begin{aligned}
& a_{1}=\beta\left(\mathscr{c}_{1}\right) \\
& \beta\left(\mathscr{L}_{1}\right)=a_{1} \\
& a_{2}=\beta\left(\mathscr{c}_{2}\right)-\mathrm{s}_{1} \\
& \text { If } a_{1} \cap a_{2} \neq \emptyset \text { then } a_{2}=a_{2} \cup a_{1} \\
& \beta\left(\mathscr{q}_{2}\right)=a_{2}
\end{aligned}
$$

3. For $\mathrm{j}=3,4, \ldots, \mathrm{n}$ do

3.1 If $\mathrm{s}_{\mathrm{j}-1} \neq \mathrm{s}_{\mathrm{j}-2}$ then do

$$
\begin{aligned}
& \text { For } k=1,2, \ldots, j-1 \text { do } \\
& \text { If } k \notin s_{j-1} \text { then do } \\
& a_{k}=\beta\left(\mathscr{G}_{\mathrm{k}}\right)-\mathrm{s}_{\mathrm{j}-1} \\
& \text { For } i=1,2, \ldots, \mathrm{k}-1 \text { do } \\
& \text { If } i \notin \mathrm{s}_{\mathrm{j}-1} \text { and } a_{\mathrm{k}} \cap a_{i} \neq \emptyset \text { then } a_{\mathrm{k}}=a_{\mathrm{k}} \cup a_{i}
\end{aligned}
$$

3.2 If $a_{j}=\beta\left(\sigma_{i}\right)-s_{j-1}$ 
3.3 For $i=1,2, \ldots, j-1$ do

If $i \notin \mathrm{s}_{\mathrm{j}-1}$ and $\alpha_{\mathrm{j}} \cap \alpha_{\mathrm{i}} \neq \emptyset$ then $\alpha_{\mathrm{j}}=\alpha_{\mathrm{j}} \cup \alpha_{\mathrm{i}}$

$3.4 \beta\left(\mathcal{Q}_{\mathrm{j}}\right)=a_{\mathrm{j}}$

We illustrate this algorithm with the pattern $\mathscr{b}$ of example (4.5). The only nonempty Hall set $S_{j}, 1 \leq j \leq 4$, is $S_{4}=\left\{\mathscr{b}_{1}, \mathscr{b}_{4}\right\}$ giving $\mathrm{s}_{4}=\{1,4\}$. For $\mathrm{j}=4, \mathrm{~s}_{2}=\mathrm{s}_{3}=\emptyset$, so computation of $2_{4}$ proceeds from step 3 of algorithm 4.10 as follows. In step $3.2, a_{4}=\beta\left(\mathscr{b}_{4}\right)=\{4\}$. In step 3.3 , for $i=1,2,3$, accumulate $\beta\left(\mathscr{c}_{1}\right), \beta\left(\mathscr{\swarrow}_{2}\right), \beta\left(\mathscr{K}_{3}\right)$ into $\alpha_{4}$, giving $\alpha_{4}=\{1,2,3,4,5,6\}$, and so $2_{4}$ is full. For $\mathrm{j}=5$, as $\mathrm{s}_{4} \neq \mathrm{s}_{3}$ in step 3.1 , update $a_{2}=\{2,6\}$ and $a_{3}=\{3,5\}$. In step $3.2, a_{5}=\beta\left(\mathscr{b}_{5}\right)-\mathrm{s}_{4}=\{3,5\}$. This remains unchanged in step 3.3 because now $a_{5} \cap a_{2}=\emptyset$ and $a_{5} \cap a_{3}=a_{5}$. Thus step 3.4 gives $\beta\left(q_{5}\right)=\alpha_{5}=\{3,5\}$. The entire pattern 2 is given by

$$
2=\left[\begin{array}{lllll}
* & * & * & * & 0 \\
0 & * & * & * & 0 \\
0 & 0 & * & * & * \\
* & * & * & * & 0 \\
0 & 0 & * & * & * \\
0 & * & * & * & 0
\end{array}\right]
$$

We now summarize a graph theoretic way to compute the Hall sets required by step 1 of algorithm 4.10. Firstly, a maximal transversal in the $m-b y-n$ pattern $\mathscr{B}$ is obtained; see, e.g. [LPP, p. 15] for an aigorithm, or $[\mathbb{D}]$ for the case $m=n$. Hithout loss of generality, this maximum matching gives a square $n-b y-n$ pattern in which all diagonal entries are *. Secondly, for the digraph associated with this square pattern, find the strongly connected components using an algorithm of Tarjan (see, e.g. [RND]), and use these to construct its condensation digraph. The Hall sets of maximal cardinality can 
be determined from this condensation digraph by traversing paths starting at vertices of indegree zero.

The time complexity of this procedure to determine the Hall sets is dominated by the computation of a maximal transversal, and is $0\left(\tau(m+n)^{\frac{1}{2}}\right)$, where $\tau$ is the number of nonzero entries in $\mathscr{6}$ (see, e.g. [LP]). Step 3 of algorithm 4.10 has time complexity $O\left((h+1) \mathrm{mn}^{2}\right)$ where $h$ is the number of distinct (nonempty) maximal Hall sets $S_{j}, 1 \leq j \leq n-1$. 


\section{Pattern $\mathscr{R}$.}

Given a pattern $\mathscr{G}$, theorem 4.7 determines the pattern 2 , and we now consider the pattern $R$. As noted in the introduction, the pattern $\mathscr{R}$ is invariant under row permutations of $\mathscr{6}$

Theorem 5.1. For $\mathrm{m} \geq \mathrm{n}$, let $\mathscr{6}$ be an $\mathrm{m}$-by-n pattern with the Hall property. If the pattern 2 is determined as in theorem 4.7 , then the pattern $\mathscr{R}$ is given by the upper triangular part of $\mathscr{2}^{\mathrm{T}} \mathscr{K}$.

Proof: Without loss of generality, we can assume that each diagonal position of $\mathscr{B}$ is *. Since $\mathrm{i} \in \mathrm{p}_{\mathrm{i}}, 1 \leq \mathrm{i} \leq \mathrm{n}$, the $(\mathrm{i}, \mathrm{i})$ entry of $\mathcal{L}$ is $*$ by theorem 4.7. Thus, since the (i, i) entry of $\mathscr{b}$ is $*$, the (i, i) entry of $\mathscr{2}^{T} \mathscr{C}$ is *. The diagonal entries of $R$ are all * (by definition of the unique $Q R$ factorization), thus the patterns of $\mathscr{L}^{\mathbb{T}} \mathscr{b}$ and $\mathscr{R}$ agree on the diagonal.

Now consider in $\mathscr{R}$ a fixed $(i, j)$ entry with $i<j \leq n$. If $\mathscr{2}_{i}$ is combinatorially orthogonal to $\mathscr{C}_{\mathrm{j}}$, then $\mathscr{2}_{\mathrm{i}}^{\mathrm{T}} \mathscr{C}_{\mathrm{j}}=0$. Also, for any $\mathrm{A} \in \mathscr{K}$, if $A=Q R$, then $R=Q^{T} A$ and so $r_{i j}=Q_{i}^{T} A_{j}=0$ in this case of combinatorial orthogonality.

If $\mathscr{2}_{i}$ is not combinatorially orthogonal to $\mathscr{C}_{\mathrm{j}}$, then $\mathscr{2}_{i}^{T} \mathscr{C}_{\mathrm{j}}$ is *. We must show that there exists a matrix $A \in \mathscr{G}$ such that if $A=Q \mathbb{R}$, then $\vec{I}_{i j} \neq 0$. Süppose that the $(k, j)$ entry of $\sqrt{b}$ is $*$ for some $k$, $1 \leq \mathrm{k} \leq \mathrm{m}$, and the $(\mathrm{k}, \mathrm{i})$ entry of $\mathcal{2}$ is *. Then $\mathrm{k} \in \mathrm{p}_{i}$, and by the construction in theorem 4.4, the submatrix in the first $i$ columns of $A$ can be determined so that in its $Q R$ factorization, the entry $q_{k i} \neq 0$. (Note that in the $Q R$ factorization of any $A \in \mathscr{K}, Q_{i}$ is completely determined by the first $i$ columns of A.) Since $\mathscr{Q}_{i}$ and $\mathscr{G}_{j}$ are not combinatorially 
orthogonal, we may choose $A_{j} \in \mathscr{b}_{j}$ so that $A_{j}$ is not orthogonal to the already determined $Q_{i}$. Thus $r_{i j}=Q_{i}^{T} A_{j}$ is nonzero, completing the proof. (Note that only columns $A_{1}, \ldots, A_{i}$ and $A_{j}$ have been specified; the other columns of $A$ are arbitrary subject to $A \in \mathscr{6}$.)

Returning again to example (1.1), we see that theorem 4.7 predicts the true pattern for 2, that is as in (1.2) with $\otimes=0$. Theorem 5.1 then predicts the correct pattern for $\mathscr{R}$, again (1.2) with $\otimes=0$. For example, the $(4,6)$ entry of $R$ is zero, as $2_{4}$ is combinatorially orthogonal to $\mathscr{K}_{6}$.

The $(i, j)$ entry of $R$ is * if and only if there is an index $k$ such that $a_{k j} \neq 0$, and a path in $B_{i}(\mathscr{C})$ from column $i$ to row $k$ (passing only through columns numbered less than i). If $\mathscr{6}$ has the strong Hall property and $A \in \mathscr{C}$, this is equivalent (ignoring cancellation) to the existence of a path in the graph of $A^{T}$ from $i$ to $j$ passing only through vertices less than i. As the existence of such a path is equivalent to a generically nonzero entry in the $(i, j)$ position of $U$ in the $L U$ factorization of $\mathrm{A}^{\mathrm{T}} \mathrm{A}$, our result for $\Omega$, specialized to the strong Hall case, yields the same result as that of [CEG] mentioned in our introduction. We note that, since the columns of 2 are determined sequentially (without appeal to previously determined columns), the rows of $\Omega$ may be determined seriaily. Thus, if only the pattern of $\mathscr{A}$ is of interest, it may be determined without necessity for storage of 2. In fact, if only row $i$ of $\Re$ is desired, it may be determined from $2_{i}$ (and $\mathscr{b}$ ) for which only the Hall set $S_{i-1}$ and $\mathscr{B}_{i}(\mathscr{B})$ are necessary. 
Remarks:

We thank F. Ruskey, M. Gillespie and the referees for useful comments. We also note that lemma 2.5 of [Gi] (which was brought to our attention after preparation of this manuscript) is closely related in substance to our theorem 3.2. The two proofs are quite different, and the underlying concept seems to be an important one for exhibiting nonzeros in factorizations of sparse matrices. The report [Gi] does not consider strong Hall critical matrices, as in our theorem 3.1 .

In addition, A. Pothen [private communication in response to our manuscript] proposed an alternate proof of our theorem 4.4, based on the Dulmage-Mendelsohn decomposition and a statement equivalent to lemma 2.5 of [Gi]. Pothen also notes that the DII decomposition can be used to construct the required Hall sets, and notes (via an argument based on algebraic indeterminates) that the collection of all entries that may individually be nonzero in $Q$ may simultaneously be nonzero for some (in fact, almost all) allowed instances of $A$. 


\section{References}

[CEG] T. Coleman, A. Edenbrandt and J. Gilbert, Predicting fill for sparse orthogonal factorization, J. Assoc. for Comp. Mach. 33(1986), $517-532$.

[D] I.S. Duff, On algorithms for obtaining a maximum transversal, ACM Transactions on Mathematical Software 7(1981), 315-330.

[Ge] W.H. Gentleman, Row elimination for solving sparse linear systems and least squares problems, in Proc. of the 1975 Dundee Conference on Numerical Analysis, G.A. Watson (ed.), Lecture Notes in Mathematics 506, Springer-Verlag, New York, 1976, 122-133.

[GH] A. George and H. Heath, Solution of sparse linear least squares problems using Givens rotations, LAA 34(1980), 69-83.

[GLN] A. George, J. Liu, and E. Ng, Row-ordering schemes for sparse Givens transformations I: Bipartite Graph Model, LAA 61(1984), 55-81.

[GN] A. George and E. Ng, Symbolic factorization for sparse Gaussian elimination with partial pivoting, SIAM J. Sci. Stat. Comput. $8(1987), 877-898$.

[Gi] J.R. Gilbert, An efficient parallel sparse partial pivoting algorithm, Chr. Michelsen Inst. Dept. of Science and Technology Report 88/45052-1, Bergen, Norway (1988).

[HJ] R. Horn and C.R. Johnson, Matrix Analysis, Cambridge Univ. Press, N.Y., 1985 .

[JOD] C.R. Johnson, D.D. Olesky and P. van den Driessche, Inherited matrix entries: LU factorizations, SIAM J. of Matrix Analysis 10(1989), 94-104.

[LP] L. Lovász and M.D. Plummer, Matching Theory, North-Holland, Amsterdam, 1986.

[RND] E.H. Reingold, J. Nievergelt and N. Deo, Combinatorial Algorithms: Theory and Practice, Prentice-Hall, Inc., New Jersey, 1977.

[S] G.W. Stewart, Perturbation bounds for the QR factorization of a matrix. SIAII J. Numer. Anal. 14(1977), 509-518. 